\title{
Strain-spectroscopy of strongly interacting defects in superconducting qubits
}

\author{
Octavio de los Santos-Sánchez $\oplus^{1}$ and Ricardo Román-Ancheyta $\circledast^{2, *}$ \\ ${ }^{1}$ Escuela de Ingeniería y Ciencias, Instituto Tecnológico y de Estudios Superiores de Monterrey, \\ Avenida San Carlos 100, Campus Santa Fe, Ciudad de México, 01389, Mexico \\ ${ }^{2}$ Instituto Nacional de Astrofísica, Óptica y Electrónica, \\ Calle Luis Enrique Erro 1, Sta. Ma. Tonantzintla, Puebla CP 72840, Mexico
}

(Dated: November 16, 2021)

\begin{abstract}
The proper functioning of some micro-fabricated novel quantum devices, such as superconducting resonators and qubits, is severely affected by the presence of parasitic structural material defects known as tunneling two-level-systems (TLS). Recent experiments have reported unambiguous evidence of the strong interaction between individual (coherent) TLS using strain-assisted spectroscopy. This work provides an alternative and simple theoretical insight that illustrates how to obtain the spectral response of such strongly interacting defects residing inside the amorphous tunnel barrier of a qubit's Josephson junction. Moreover, the corresponding spectral signatures obtained here may serve to quickly and efficiently elucidate the actual state of these interacting TLS in experiments based on strain- or electric-field spectroscopy.
\end{abstract}

\section{INTRODUCTION}

Impressive progress in the field of circuit quantum electrodynamics has occurred since the first realization of the coherent interaction between a superconducting qubit with a single microwave photon [1]. To date, such a coupled system constitutes the building block for the state-of-the-art prototypes of solid-state-based quantum processors [2]. Unfortunately, it is still challenging to preserve the qubits' coherence for a long time due to the unavoidable interaction with their environment $[3,4]$, including cosmic rays $[5,6]$. A dominant source of decoherence is the random formation of natural defects at circuit interfaces and inside the Josephson junctions (JJs), whereby a qubit is highly sensitive [7-10].

Superconducting qubits must operate at low temperatures, a condition under which amorphous solids, such as the typical oxide tunnel barrier of a JJ, exhibit a universal behavior explained by the so-called standard tunneling model (STM) [11]. This phenomenological model describes atomic-scale defects in amorphous materials that have long been construed as tunneling two-level systems (TLS) $[12,13]$. The presence of TLS affects, severely, the frequency range of operation and coherence time of superconducting qubits $[8,14,15]$, so that several strategies to improve the qubits' quality have been implemented [16-18]; for instance, the use of JJs of small area [9]. Nevertheless, the sensitivity to TLS makes superconducting qubits fitting devices to investigate individual TLS [19] in virtually arbitrary materials [20] and, hopefully, to be able to reveal their true microscopic origin [21]. On the other hand, earlier proposals used TLS as logical qubits [22] or quantum memories, as experimentally demonstrated in [23]

The possibility of the occurrence of strong interactions between TLS, formerly surmised in [24], has already been

\footnotetext{
*ancheyta6@gmail.com
}

confirmed via a novel experimental technique for highresolution strain-tuning spectroscopy [25]. In this context, through bending a qubit chip sample, defect spectroscopy data showed the distinctive avoided level crossings embedded in a very peculiar S-shaped signal [25]. More recently, with the same technique, electric fields were used to find the precise location of individual TLS on the chip circuit [26, 27]. A laborious task in these types of experiments is to search for and identify authentic signatures of mutual TLS interactions from the intricate plots of their spectroscopic data [20, 28, 29]. Due to such difficulties, the present work aims to provide a theoretical description of some spectroscopic measurements associated with interacting TLS.

In this work, we use the method of small rotations $[30,31]$ to get an effective Hamiltonian that accurately describes the energy level structure of two strongly interacting TLS residing in the tunnel barrier of a qubit's JJ. We also propose using the Eberly-Wódkiewicz (EW) spectrum [32] as an alternative way to obtain the spectral response of those interacting TLS. In contrast to the standard Wiener-Khintchine power spectrum, computing the EW spectrum is more straightforward and does not require knowledge of a stationary state [33]. The EW spectrum has recently been used to describe ultrastrongly coupled quantum systems [34], intermittent resonance fluorescence [35], and signatures of non-thermal baths in quantum thermalization processes [36]. Here, we obtain explicit expressions for the typical spectra of two coherently interacting TLS in qubit chips where strain or electric fields are applied. Our results show that the EW spectrum reproduces, reasonably well, the behavior of previously reported strain-spectroscopic data, including the characteristic S-shaped signal.

The structure of the paper is as follows. In Sec. II, we make a brief description of the STM and present the Hamiltonian of two interacting TLS in the tunnel barrier of a qubit's JJ. In Sec. III, we implement a unitary transformation to obtain an effective Hamiltonian with simplified eigenvalues and eigenstates. In Sec. IV, we evaluate 
the EW spectrum and obtain the spectral response of the corresponding coupled TLS. Finally, Sec. V outlines our conclusions.

\section{MODEL}

Our description is based upon the STM, whose visualization is that of an atom that can be sit in one of two parabolic potentials of a double-well, as depicted in Fig. 1. Initially conceived by Phillips [37] and Anderson [38] in the context of amorphous solids, the model is such that the minima of the wells represent energetically similar metastable states of an atom that are accessible via coherent tunneling. The inter-well tunneling energy $\Delta$ captures the capability of the atom to tunnel the potential barrier, and $\varepsilon$ is the energy asymmetry associated with the corresponding ground-state wavefunctions of the wells. Having specified the aforesaid atomic TLS parameters, which vary from defect to defect in amorphous solids [21], the evolution of a single tunneling atom is ruled by the Hamiltonian (in units of $\hbar=1$ ) [37, 38]

$$
H_{T L S}=\frac{1}{2} \varepsilon \sigma_{z}+\frac{1}{2} \Delta \sigma_{x},
$$

where $\sigma_{z}=|R\rangle\langle R|-| L\rangle\langle L|$ and $\sigma_{x}=|R\rangle\langle L|+| L\rangle\langle R|$ are the Pauli matrices written in the position or coordinate basis comprising of the left $(|L\rangle)$ and right $(|R\rangle)$ state vectors. The eigenstates of the Hamiltonian above, henceforth labeled as excited $(|e\rangle)$ and ground $(|g\rangle)$ states, are, respectively, $|e\rangle=\sin (\theta / 2)|L\rangle+\cos (\theta / 2)|R\rangle$, and $|g\rangle=\cos (\theta / 2)|L\rangle-\sin (\theta / 2)|R\rangle$, with $\tan \theta=\Delta / \varepsilon$. Within this diagonal basis, Hamiltonian (1) boils down to the simple form

$$
H_{T L S}=\frac{1}{2} \omega \tilde{\sigma}_{z},
$$

where the tilde denotes Pauli operators in the eigenbasis and $\omega$ is the transition energy $E=E_{e}-E_{g}$ (also depicted in Fig. 1) between eigenstates

$$
\omega=\sqrt{\varepsilon^{2}+\Delta^{2}} .
$$

Experimentally, the asymmetry energy $\varepsilon$ may be linearly driven by mechanical strain on the qubit chip, which, in turn, can be manipulated at will via an applied piezo voltage $V_{p}$ [21]; i.e., $\varepsilon=\varepsilon\left(V_{p}\right)$ and, needless to say, the energy splitting will also be dependent on the strain field $\omega=\omega\left(V_{p}\right)$. Strain tuning experiments in [19] confirm the hyperbolic dependence of (3) on the asymmetry energy $\varepsilon$, providing firm evidence for a central hypothesis that TLS are the cause of avoided level crossings in the frequency of JJ qubits.

Strain interactions between TLS occur by exchanging phonons $[39,40]$. In order to go into the scenario of two interacting TLS (labeled as TLS1 and TLS2) residing in the tunnel barrier of a JJ, the following Hamiltonian has been posited by Lisenfeld et al. [25] $H_{T}=$ (a)

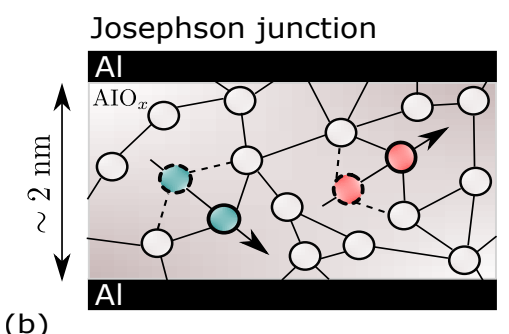

(b)

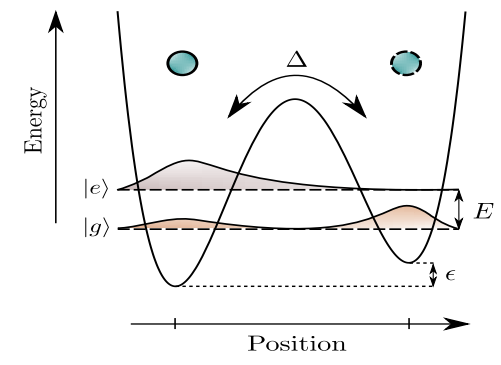

FIG. 1. (a) Sketch of a Josephson tunnel junction consisting of two $\mathrm{Al}$ electrodes (black thin rectangles) isolated by a $\mathrm{AlO}_{x}$ (amorphous aluminum oxide) dielectric layer with a typical thickness of $\sim 2-3 \mathrm{~nm}$. The latter hosts two defects viewed as atoms tunneling between two metastable locations (green and red circles); the arrows denote their dipole moment. (b) Double-well potential model corresponding to a single atom tunneling between two metastable states. Tunneling and asymmetry energies, $\Delta$ and $\varepsilon$, respectively, set the transition energy $E=E_{e}-E_{g}=\sqrt{\Delta^{2}+\varepsilon^{2}}$ between the TLS eigenstates.

$H_{1}+H_{2}+H_{\text {TLS-TLS }}$, in which $2 H_{i}=\varepsilon_{i}\left(V_{p}\right) \sigma_{z}^{(i)}+\Delta_{i} \sigma_{x}^{(i)}$ represents the Hamiltonian of a single defect $i$ and the TLS-TLS interaction, $H_{\text {TLS-TLS }}$, is taken to be described in the position basis as [21]:

$$
H_{\text {TLS-TLS }}=\frac{1}{2} g \sigma_{z}^{(1)} \sigma_{z}^{(2)},
$$

with $g$ being the strength of the defects' coupling whose value can be inferred from direct spectroscopic measurements. In this work, as in [25], we find it convenient to reframe Hamiltonian $H_{T}$ by deploying the transformation $\sigma_{z}^{(i)} \rightarrow \cos \theta_{i} \tilde{\sigma}_{z}^{(i)}+\sin \theta_{i} \tilde{\sigma}_{x}^{(i)}$ and then neglecting coupling terms of the form $\propto \tilde{\sigma}_{x}^{(1)} \tilde{\sigma}_{z}^{(2)}$ and $\propto \tilde{\sigma}_{z}^{(1)} \tilde{\sigma}_{x}^{(2)}$, because they contribute as small energy offsets. Thus, the following Hamiltonian is obtained in the diagonal basis

$$
H_{T}=\frac{\omega_{1}}{2} \tilde{\sigma}_{z}^{(1)}+\frac{\omega_{2}}{2} \tilde{\sigma}_{z}^{(2)}+\frac{g_{\|}}{2} \tilde{\sigma}_{z}^{(1)} \tilde{\sigma}_{z}^{(2)}+\frac{g_{\perp}}{2} \tilde{\sigma}_{x}^{(1)} \tilde{\sigma}_{x}^{(2)} .
$$

Here, $\omega_{i}=\sqrt{\varepsilon_{i}^{2}\left(V_{p}\right)+\Delta_{i}^{2}}, i=1,2 ; g_{\|}=g \cos \theta_{1} \cos \theta_{2}$ and $g_{\perp}=g \sin \theta_{1} \sin \theta_{2}$ are referred to as, respectively, the longitudinal and transversal coupling components, with $\cos \theta_{i}=\varepsilon_{i} / \omega_{i}$ and $\sin \theta_{i}=\Delta_{i} / \omega_{i}$. So, each TLS is characterized by the tunneling energy $\Delta_{i}$ and the strain-dependent asymmetry energy $\varepsilon_{i}\left(V_{p}\right)$. The third and fourth terms appearing in (5) are responsible for frequency shift and energy exchange effects, respectively, 
accounting for the spectral profile of the defect interaction already experimentally reported [25], as we will see below. Again, we remark that the asymmetry energy referred to above can be tuned by slightly bending the chip circuit using a piezo actuator, according to the rule $\varepsilon_{i}=c_{i}\left(V_{p}-V_{0 i}\right)$ [19]; $V_{0 i}$ is the voltage at which the TLS is at its symmetry point and $c_{i}$ is an adjusting parameter.

\section{ENERGY SPECTRUM}

Hamiltonian (5) has been successful in predicting, numerically, the transition energies of interacting TLS in a phase qubit [25]. Here we find that an algebraic diagonalization of it is possible, allowing us to arrive at the following closed-form expressions for its four eigenenergies:

$$
\begin{aligned}
& E_{1,2}=\mp \frac{1}{2} \sqrt{\left(\omega_{1}+\omega_{2}\right)^{2}+g_{\perp}^{2}}+\frac{g_{\|}}{2}, \\
& E_{3,4}= \pm \frac{1}{2} \sqrt{\left(\omega_{1}-\omega_{2}\right)^{2}+g_{\perp}^{2}}-\frac{g_{\|}}{2} .
\end{aligned}
$$

Associated with these eigenenergies, the corresponding eigenstates are given, in the basis $\{|e, e\rangle,|e, g\rangle,|g, e\rangle,|g, g\rangle\}$, respectively, by

$$
\begin{aligned}
& \left|u_{1}\right\rangle=\cos (\alpha / 2)|g, g\rangle-\sin (\alpha / 2)|e, e\rangle, \\
& \left|u_{2}\right\rangle=\cos (\alpha / 2)|e, e\rangle+\sin (\alpha / 2)|g, g\rangle, \\
& \left|u_{3}\right\rangle=\cos (\beta / 2)|e, g\rangle+\sin (\beta / 2)|g, e\rangle, \\
& \left|u_{4}\right\rangle=\cos (\beta / 2)|g, e\rangle-\sin (\beta / 2)|e, g\rangle,
\end{aligned}
$$

where $\tan \alpha=g_{\perp} /\left(\omega_{1}+\omega_{2}\right)$ and $\tan \beta=g_{\perp} /\left(\omega_{1}-\omega_{2}\right)$. Besides this result, one of the main contributions of this work is to remark the fact that, even in the strong coupling regime, Hamiltonian (5) can be further simplified by applying the transformation $T=e^{\eta G}$, with $G=\tilde{\sigma}_{+}^{(1)} \tilde{\sigma}_{+}^{(2)}-\tilde{\sigma}_{-}^{(1)} \tilde{\sigma}_{-}^{(2)}$ and choosing $\eta=g_{\perp} /\left[2\left(\omega_{1}+\omega_{2}\right)\right]$ $[30,31]$. Accordingly, keeping second-order terms involving $\eta$, we get the effective Hamiltonian $\left(H_{\text {eff }}=T H_{T} T^{\dagger}\right)$ :

$$
\begin{aligned}
H_{\mathrm{eff}} \approx \frac{\bar{\omega}_{1}}{2} \tilde{\sigma}_{z}^{(1)}+\frac{\bar{\omega}_{2}}{2} \tilde{\sigma}_{z}^{(2)}+\frac{g_{\|}}{2} \tilde{\sigma}_{z}^{(1)} \tilde{\sigma}_{z}^{(2)} \\
+\frac{g_{\perp}}{2}\left(\tilde{\sigma}_{+}^{(1)} \tilde{\sigma}_{-}^{(2)}+\tilde{\sigma}_{-}^{(1)} \tilde{\sigma}_{+}^{(2)}\right),
\end{aligned}
$$

where the energy exchange term, weighted by $g_{\perp}$, is now reminiscent of that of two dipole coupled two-level systems in the rotating-wave approximation, and we have defined

$$
\bar{\omega}_{j}=\omega_{j}+\frac{g_{\perp}^{2}}{4\left(\omega_{1}+\omega_{2}\right)} .
$$

So, the four eigenvalues obtained from this effective Hamiltonian take the form

$$
\begin{aligned}
E_{1,2}^{\mathrm{eff}} & =+\frac{g_{\|}}{2} \mp \frac{1}{2}\left(\bar{\omega}_{1}+\bar{\omega}_{2}\right), \\
E_{3,4}^{\mathrm{eff}} & =-\frac{g_{\|}}{2} \pm \frac{1}{2} \sqrt{\left(\bar{\omega}_{1}-\bar{\omega}_{2}\right)^{2}+g_{\perp}^{2}} .
\end{aligned}
$$

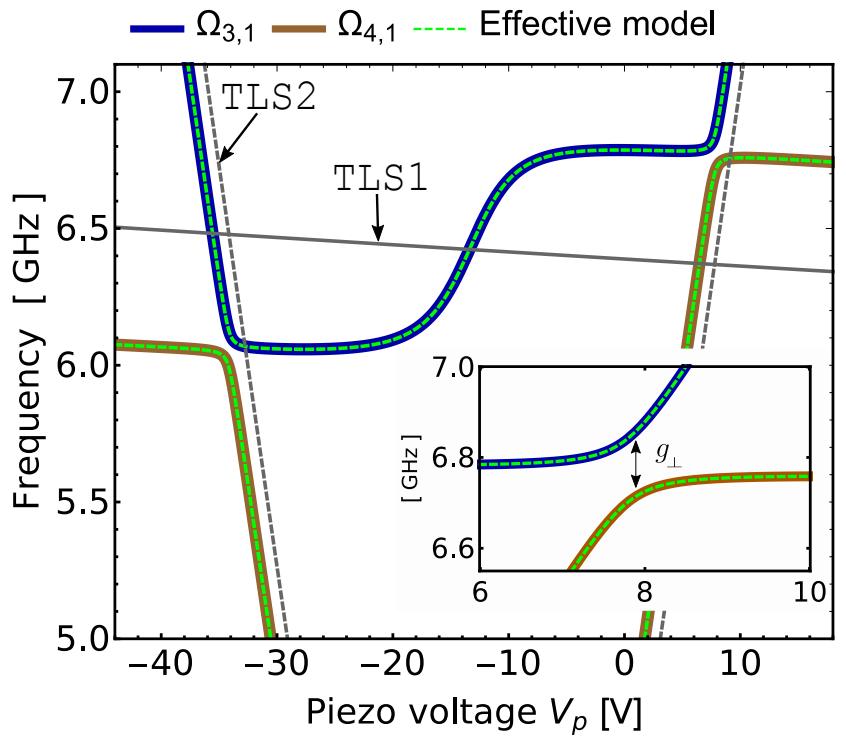

FIG. 2. Energy spectrum, $\Omega_{k, 1}=E_{k}-E_{1}$, with $k=3$, 4 , obtained by diagonalizing the total (5) and effective (8) Hamiltonian, vs. the piezo voltage, $V_{p}$. Gray lines represent the bare frequency of TLS1 (continuous) and TLS2 (dashed). System parameters: $g=800 \mathrm{MHz}, \Delta_{1}=5.47 \mathrm{GHz}$ and $\Delta_{2}=1.3$ $\mathrm{GHz} ; \varepsilon_{1}=c_{1} V_{p}-3.3[\mathrm{GHz}]$ and $\varepsilon_{2}\left(V_{p}\right)=c_{2}\left(V_{p}+13[\mathrm{~V}]\right) ; c_{1}=5$ $\mathrm{MHz} / \mathrm{V}$ and $c_{2}=0.3 \mathrm{GHz} / \mathrm{V}$. The inset zooms in on the vicinity of the right avoided level crossing where $V_{p} \sim 7.78 \mathrm{~V}$, $g_{\perp} \sim 140 \mathrm{MHz}$ and $g_{\|} \sim-401 \mathrm{MHz}$.

Correspondingly, the eigenstates are also simplified as follows:

$$
\begin{aligned}
& \left|u_{1}\right\rangle_{\mathrm{eff}}=|g, g\rangle, \\
& \left|u_{2}\right\rangle_{\mathrm{eff}}=|e, e\rangle, \\
& \left|u_{3}\right\rangle_{\mathrm{eff}}=\cos (\beta / 2)|e, g\rangle+\sin (\beta / 2)|g, e\rangle, \\
& \left|u_{4}\right\rangle_{\mathrm{eff}}=\cos (\beta / 2)|g, e\rangle-\sin (\beta / 2)|e, g\rangle .
\end{aligned}
$$

In Fig. 2, we display a comparison between the outcome of the total Hamiltonian (5) (continuous lines) and that of the effective one (8) (green dashed lines) by plotting the strain dependence of the transition energies $\Omega_{k, 1} \equiv E_{k}-E_{1}$, for $k=3,4$, making use of Eqs. (6a)-(6b) and (10a)-(10b). The transition energies may occur between the entangled states, $\left|u_{3}\right\rangle_{\text {eff }}$ and $\left|u_{4}\right\rangle_{\text {eff }}$, with the state $\left|u_{1}\right\rangle_{\text {eff }}$. This identification permits us to assess, for instance, the transversal coupling $g_{\perp}$ that takes part in the defect interplay: near the anti-crossing region, $\omega_{1} \sim \omega_{2}$ (i.e., at resonant locus where the avoided level crossings take place) spectroscopic techniques enable one to trace the energy splitting $\Omega_{3,1}-\Omega_{4,1}=\left[\left(\omega_{1}-\omega_{2}\right)^{2}+g_{\perp}^{2}\right]^{1 / 2} \sim g_{\perp}$. The system parameters used in the present calculation are taken to be close to the data formerly reported [25]: the interplay coupling strength $|g|=800 \mathrm{MHz}$; as to tunneling energies, $\Delta_{1}=5.47 \mathrm{GHz}, \Delta_{2}=1.3 \mathrm{GHz}$; the asymmetry energies are such that $\varepsilon_{1}=c_{1} V_{p}-3.3[\mathrm{GHz}]$ and $\varepsilon_{2}\left(V_{p}\right)=c_{2}\left(V_{p}+13[\mathrm{~V}]\right)$, with $c_{1}=5 \mathrm{MHz} / \mathrm{V}$ and $c_{2}=0.3$ $\mathrm{GHz} / \mathrm{V}$; the choice of these parameters is such that, in the 
displayed domain, TLS1 is barely affected by the mechanical strain. With the help of the foregoing information, we get $g_{\perp} \sim 0.14 \mathrm{GHz}$, the two avoided level crossings are found to be positioned at $V_{p, \pm} \sim\{-34.15,7.78\}[V]$, and, say, at the right resonant location, the normalized coupling $g_{\perp} / \omega_{1,2} \sim 0.022$ is obtained; this makes legitimate the use of the effective Hamiltonian (8) conserving solely the rotating terms [41]. So, regarding the energy spectrum, we confirm that our effective model (green dashed lines) makes essentially the same prediction as the total Hamiltonian (dark blue and brown solid lines). In the next section, $H_{\text {eff }}$ will be helpful in obtaining the spectral response of the interacting TLSs.

\section{STRAIN-DEPENDENT SPECTRA}

Customarily, one would describe the system's open dynamics under study by establishing a fitting local [42], or global [43], master equation. From this standpoint, knowledge of the steady-state of the system would be necessary to compute, for instance, the stationary power spectrum à la Wiener-Khintchine to attempt to trace its spectroscopy. However, as far as we know, information about the intrinsic damping mechanisms within amorphous materials involved in mutual TLS coherent interactions is neither obvious [44, 45] nor accessible, making it quite challenging to posit an accurate microscopic master equation treatment [46]. In order to sidestep this issue, we resort to carrying out a spectral measurement based upon the operational definition of spectrum for nonstationary processes, i.e., the so-called time-dependent "physical spectrum" of Eberly and Wódkiewicz [32]. In fact, the EW spectrum has recently and successfully described the ultrastrong coupling between light and matter [34] and even quantum thermalization processes [36]. Remarkably, the EW spectrum will also permit us to concisely illustrate the spectral response of strongly interacting TLS in experiments based on strain-field spectroscopy. The standard definition of it is given by $[32,47]$

$$
\begin{aligned}
S(\omega, \Gamma, t)=2 \Gamma \int_{0}^{t} d t_{1} \int_{0}^{t} d t_{2} e^{-(\Gamma+i \omega)\left(t-t_{2}\right)} \\
\times e^{-(\Gamma-i \omega)\left(t-t_{1}\right)} G\left(t_{1}, t_{2}\right),
\end{aligned}
$$

where $\Gamma$ is the band half-width of a given interferometer acting as a filter and $\omega$ its central frequency. $G\left(t_{1}, t_{2}\right)$ is the corresponding two-time autocorrelation function that for the $i^{\text {th }}$ TLS reads $G\left(t_{1}, t_{2}\right)=\left\langle\tilde{\sigma}_{+}^{(i)}\left(t_{1}\right) \tilde{\sigma}_{-}^{(i)}\left(t_{2}\right)\right\rangle$, where the expectation value $\langle\cdots\rangle$ is taken with respect to some initial state of the two coupled TLS, and $\tilde{\sigma}_{ \pm}^{(i)}\left(t_{j}\right)=$ $\exp \left(i H_{T} t_{j}\right) \tilde{\sigma}_{ \pm}^{(i)} \exp \left(-i H_{T} t_{j}\right)$. Having diagonalized the total Hamiltonian (5), one can obtain a closed-form expression for $\tilde{\sigma}_{ \pm}\left(t_{j}\right)$ and, consequently, for $G\left(t_{1}, t_{2}\right)$.

If we assume that the initial state of the composite system is $|e, g\rangle=|e\rangle \otimes|g\rangle$, the autocorrelation function takes the form

$$
\begin{aligned}
G_{e g}\left(t_{1}, t_{2}\right)= & \left\langle e, g\left|\tilde{\sigma}_{+}^{(1)}\left(t_{1}\right) \tilde{\sigma}_{-}^{(1)}\left(t_{2}\right)\right| e, g\right\rangle, \\
= & \cos ^{2}(\alpha / 2) \cos ^{4}(\beta / 2) e^{i \Omega_{3,1}\left(t_{1}-t_{2}\right)} \\
& +\cos ^{2}(\alpha / 2) \sin ^{4}(\beta / 2) e^{i \Omega_{4,1}\left(t_{1}-t_{2}\right)} \\
& +\sin ^{2}(\alpha / 2) \cos ^{4}(\beta / 2) e^{i \Omega_{3,2}\left(t_{1}-t_{2}\right)} \\
& +\sin ^{2}(\alpha / 2) \sin ^{4}(\beta / 2) e^{i \Omega_{4,2}\left(t_{1}-t_{2}\right)} \\
& +\cos ^{2}(\alpha / 2) \sin ^{2}(\beta) e^{i\left(\Omega_{3,1} t_{1}-\Omega_{4,1} t_{2}\right)} / 4 \\
& +\cos ^{2}(\alpha / 2) \sin ^{2}(\beta) e^{i\left(\Omega_{4,1} t_{1}-\Omega_{3,1} t_{2}\right)} / 4 \\
& +\sin ^{2}(\alpha / 2) \sin ^{2}(\beta) e^{i\left(\Omega_{3,2} t_{1}-\Omega_{4,2} t_{2}\right)} / 4 \\
& +\sin ^{2}(\alpha / 2) \sin ^{2}(\beta) e^{i\left(\Omega_{4,2} t_{1}-\Omega_{3,2} t_{2}\right)} / 4 .
\end{aligned}
$$

Here, the subscript "eg" indicates that TLS1 is initially excited and the TLS2 is in its ground state. Superscript "(1)" above the spin operators denotes that the autocorrelation and the EW spectrum are, in this case, associated with the TLS1. Notice that the time-dependent functions in (13) are just simple exponentials; thus, their corresponding time integrals will be straightforward. So, on substitution of (13) into (12), once the transient process has elapsed, i.e., in the long time limit $\Gamma t \gg 1$ [35], this filtering integration yields the following expression for the TLS1's physical spectrum

$$
\begin{aligned}
& S_{e g}^{(1)}(\omega)= \\
& \frac{2 \Gamma \cos ^{2}(\alpha / 2) \cos ^{4}(\beta / 2)}{\Gamma^{2}+\left(\omega-\Omega_{3,1}\right)^{2}}+\frac{2 \Gamma \cos ^{2}(\alpha / 2) \sin ^{4}(\beta / 2)}{\Gamma^{2}+\left(\omega-\Omega_{4,1}\right)^{2}} \\
& +\frac{2 \Gamma \sin ^{2}(\alpha / 2) \cos ^{4}(\beta / 2)}{\Gamma^{2}+\left(\omega-\Omega_{3,2}\right)^{2}}+\frac{2 \Gamma \sin ^{2}(\alpha / 2) \sin ^{4}(\beta / 2)}{\Gamma^{2}+\left(\omega-\Omega_{4,2}\right)^{2}},
\end{aligned}
$$

with $\Omega_{k, 1 / 2} \equiv E_{k}-E_{1 / 2}(k=3,4)$ and the $E_{i}$ 's being the eigenvalues associated with the total Hamiltonian (the superscripts distinguish between TLS1 or TLS2). This result is valid to all orders of mutual TLS's coupling $g$, thereby giving rise to four components in the spectrum. Nevertheless, observation of numerical results reveals that, for the parameter regime and strain domain used in this discussion, $\cos ^{2}(\alpha / 2) \sim 1$, and the last two terms can be negligible since $\sin ^{2}(\alpha / 2) \sim 0$. Thus, the foregoing result contracts to

$$
S_{e g}^{(1)}(\omega) \approx \frac{2 \Gamma \cos ^{4}(\beta / 2)}{\Gamma^{2}+\left(\omega-\Omega_{3,1}\right)^{2}}+\frac{2 \Gamma \sin ^{4}(\beta / 2)}{\Gamma^{2}+\left(\omega-\Omega_{4,1}\right)^{2}} .
$$

These two prevailing spectral components are also predicted by our effective model (8), leading to essentially the same result. Indeed, following the procedure outlined above, and under the same initial conditions but with a much more simplified time evolution operator $\exp \left(-i H_{\text {eff }} t\right)$, yields the effective TLS1's physical spectrum:

$$
S_{e g}^{(\mathrm{eff}, 1)}(\omega)=\frac{2 \Gamma \cos ^{4}(\beta / 2)}{\Gamma^{2}+\left(\omega-\bar{\Omega}_{3,1}\right)^{2}}+\frac{2 \Gamma \sin ^{4}(\beta / 2)}{\Gamma^{2}+\left(\omega-\bar{\Omega}_{4,1}\right)^{2}},
$$




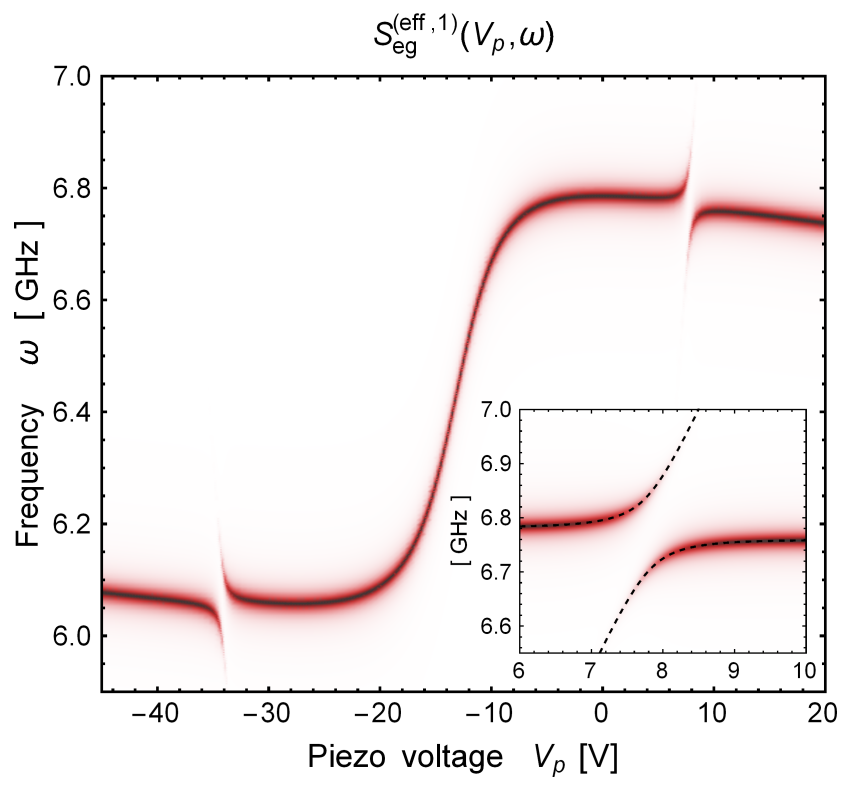

FIG. 3. Strain-dependent spectrum of TLS1 given by Eq. (16). The ordinate and abscissa indicate, respectively, the spectral detuning and piezo voltage $V_{p}$. The S-shaped profile and avoided level crossings are conspicuous. The inset zooms in on the same spectral signature in the neighbourhood of the right avoided level crossing at $\sim 7.8 \mathrm{~V}$. The initial state is taken to be the $|e, g\rangle$ state and the system parameters are the same as in Fig. 2 with $\Gamma=0.01 \mathrm{GHz}$.

where $\bar{\Omega}_{k, 1}=E_{k}^{\text {eff }}-E_{1}^{\text {eff }}$, with $E_{k}^{\text {eff }}(k=3,4)$ being given, correspondingly, by the set of effective eigenvalues (10a)-(10b). As a function of $\omega$, each of the two terms in Eq. (16) represents a Lorentzian peak of full width at half maximum (FWHM) given by $2 \Gamma$ and centered at $\bar{\Omega}_{k, 1}$. Fig. 3 displays the graphical result of the two Lorentzian line-shape functions (16) for the same system parameters as in Fig. 2. The clear evidence of the strain-dependent manifestation of mutual defect interplay is two-fold: $i$ ) We observe a clear-cut S-shaped frequency shift that stems from the longitudinal coupling, $g_{\|}$, taking part in the interaction term $\left.\propto \tilde{\sigma}_{z}^{(1)} \tilde{\sigma}_{z}^{(2)} ; i i\right)$ Two avoided level crossings come about at specific resonant positions, where the TLSs' energy exchange, caused by the interaction $\propto \tilde{\sigma}_{+}^{(1)} \tilde{\sigma}_{-}^{(2)}+$ h.c., weighted by the transversal coupling, $g_{\perp}$, is maximum (the right one is zoomed in on the right panel of the figure located at $\sim 7.8 \mathrm{~V})$. This spectral feature is consistent with the fact that the TLS1 is excited, and it is closely connected with experimental observations through swap-spectroscopy already reported in $\mathrm{AIO}_{x}$, see Fig. 3 of [25]. Unlike the energy spectrum representation depicted in Fig. 2, the outcome in Fig. 3 can be construed as a proxy for the defect's strain-dependent power spectrum. Notice that the TLS2's hyperbolic dependence (3), depicted by the gray dashed line in Fig. 2, is invisible in Fig. 3. This is because, except for the avoided level crossings, the initial excitation of the coupled system resides in the TLS1.

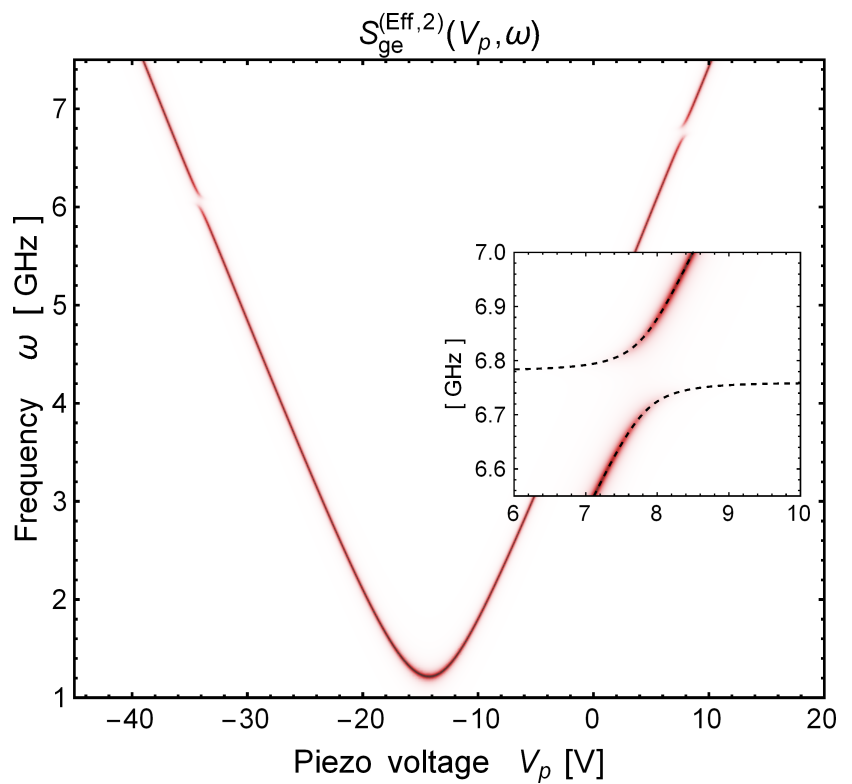

FIG. 4. Strain-dependent spectrum of TLS2 for the initial state $|g, e\rangle$, Eq. (17a). The inset spotlight the spectral profiles in the neighborhood of defect resonances at $\sim 7.8 \mathrm{~V}$. The system parameters are the same as in Fig. 2 with $\Gamma=0.021 \mathrm{GHz}$.

Admitting the possibility of being able to implement TLS2 spectrum measurements, we show in Figs. 4 and 5 the corresponding predictions as to whether the initial state of system is the $|g, e\rangle=|g\rangle \otimes|e\rangle$ or the $|e, g\rangle$ state; that is, TLS1 is in its ground state whereas TLS2 is excited, or viceversa. So, the respective spectra of TLS2 are given by

$$
\begin{aligned}
& S_{g e}^{(\mathrm{eff}, 2)}(\omega)=\frac{2 \Gamma \sin ^{4}(\beta / 2)}{\Gamma^{2}+\left(\omega-\bar{\Omega}_{3,1}\right)^{2}}+\frac{2 \Gamma \cos ^{4}(\beta / 2)}{\Gamma^{2}+\left(\omega-\bar{\Omega}_{4,1}\right)^{2}}, \\
& S_{e g}^{(\mathrm{eff}, 2)}(\omega)=\frac{\Gamma \sin ^{2}(\beta) / 2}{\Gamma^{2}+\left(\omega-\bar{\Omega}_{3,1}\right)^{2}}+\frac{\Gamma \sin ^{2}(\beta) / 2}{\Gamma^{2}+\left(\omega-\bar{\Omega}_{4,1}\right)^{2}},
\end{aligned}
$$

where $\left.\sin ^{2}(\beta)=g_{\perp}^{2} /\left[\bar{\omega}_{1}-\bar{\omega}_{2}\right)^{2}+g_{\perp}^{2}\right]$.

Depicting the TLS2's spectrum over a wider spectral detuning, we observe, in Fig. 4, the well-known hyperbolic evolution (3), with the symmetry point being discernible and located at approximately $-13 \mathrm{~V}$. Again, the interplay between TLSs manifests itself in two avoided level crossings (being located at the same positions as in the previous case); one of them, at $\sim 7.8 \mathrm{~V}$, being zoomed in on the inset of the figure. In this case, one cannot see the characteristic S-shaped signal of TLS1 because the excitation is essentially in the TLS2. Fig. 5, on the the other hand, shows the converse case in which the TLS1 is excited. In this case, we see that the TLS2's spectrum becomes conspicuous insofar as the spectral evolution between defects approach the avoided-crossing-level positions, where the resonant energy exchange is maximum. We note in passing that the feasibility of observing similar spectral features has recently been demonstrated, experimentally, via novel quantum sensors to detect TLS- 


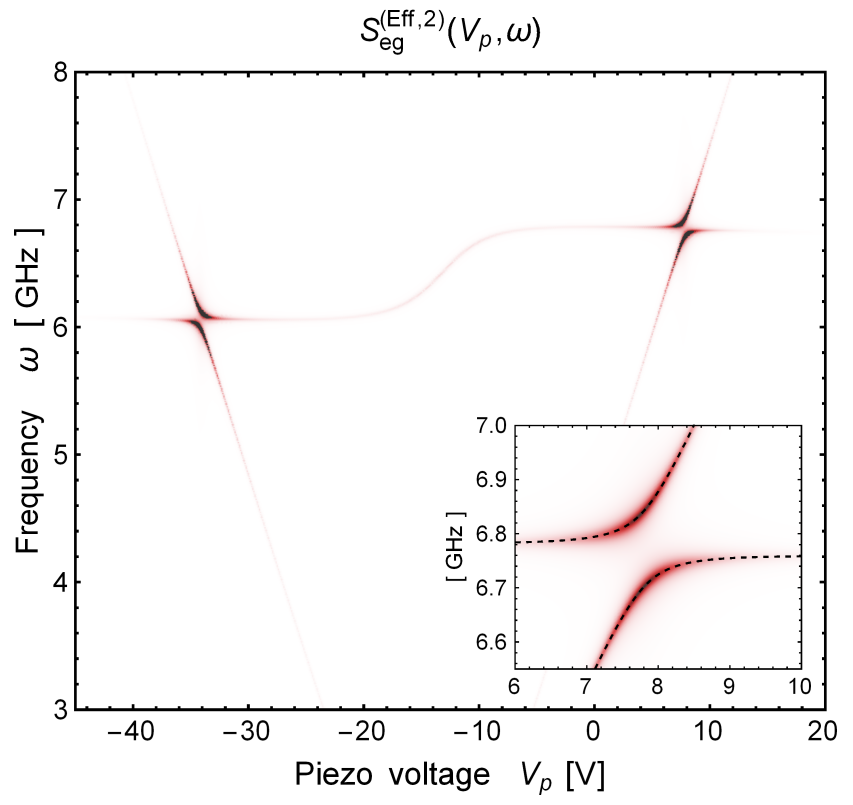

FIG. 5. Strain-dependent spectrum of TLS2 for the initial state $|e, g\rangle$, Eq. (17b). The inset spotlight the spectral profiles in the neighborhood of defect resonances at $\sim 7.8 \mathrm{~V}$. The system parameters are the same as in Fig. 2 with $\Gamma=0.01 \mathrm{GHz}$.

TLS resonances in dielectrics [20], using both applied mechanical strain and DC electric fields for characterizing their spectroscopy. For instance, Fig. 3 of [20] clearly shows segmented hyperbolic traces, as well as Supplementary Fig. 1(c) of [25].

For better understanding of the aforementioned behavior, we now discuss the role played by the weighing terms, $\cos ^{4}(\beta / 2)$ and $\sin ^{4}(\beta / 4)$, in Eq. (16), that shape the overall profile of the spectrum as a function of the piezo voltage. For instance, we see from Fig. 6 that the $\cos ^{4}(\beta / 2)$ and $\sin ^{4}(\beta / 4)$ terms are complementary to each other in Eq. (16), in the sense that they filter the spectral signal far from resonance, the combination of them giving rise to the Lorentzian-type spectrum: the first term of the spectrum, weighted by $\cos ^{4}(\beta / 2)$ (blue line), is essentially responsible for the S-shape signal (Fig. 3) to appear in the region of about $-34 \mathrm{~V} \leq V_{p} \leq 8 \mathrm{~V}$, whereas the complement trace of the spectrum, outside the aforesaid region, is weighted by the $\sin ^{4}(\beta / 4)$ term (dashed-black line) for $V_{p}<-34 \mathrm{~V}$ and $V_{p}>8 \mathrm{~V}$. The contribution of the $\sin ^{2}(\beta)$ term of Eq. (17b) (red line), on the other hand, reflects only the strong interaction between TLSs near resonance, see Fig. 5.

So far, we have concentrated on the case where the initial state contains a single excitation, $|e, g\rangle$ or $|g, e\rangle$. However, interesting results may arise if the system of two coupled TLS starts in the fully excited state $|e, e\rangle$. For the sake of simplicity, we will restrict ourselves to computing the spectral response of TLS1 only. It is easy to show that the corresponding EW spectrum, when us-

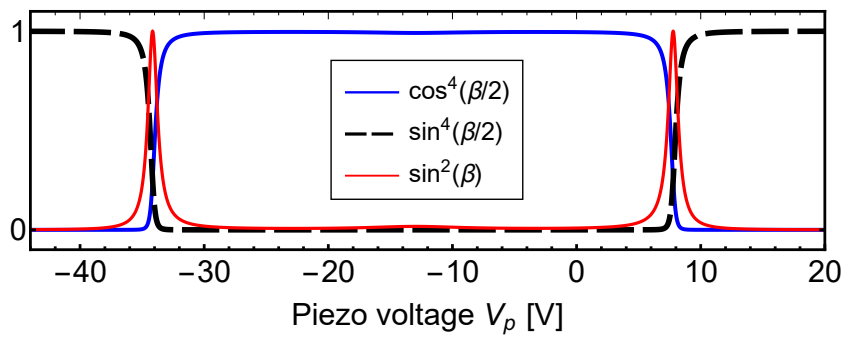

FIG. 6. Weights associated to each Lorentzian function that shape the overall profile of the physical spectrum given in Eqs. (16), (17a) and (17b).

ing the initial state $|e, e\rangle$, is

$$
S_{e e}^{(\mathrm{eff}, 1)}(\omega)=\frac{2 \Gamma \sin ^{4}(\beta / 2)}{\Gamma^{2}+\left(\omega-\bar{\Omega}_{2,3}\right)^{2}}+\frac{2 \Gamma \cos ^{4}(\beta / 2)}{\Gamma^{2}+\left(\omega-\bar{\Omega}_{2,4}\right)^{2}},
$$

where $\bar{\Omega}_{2, k}=E_{2}^{\text {eff }}-E_{k}^{\text {eff }}$. As a function of the applied piezo voltage, $S_{e e}^{(\text {eff, } 1)}(\omega)$ behaves like an inverted S-shaped signal (Z), i.e., a signal similar to that of Fig. 3 but with a left avoided level crossing located in a frequency region that is larger than the right one. This result is consistent with the experimental measurements obtained in [25] using a two-photon swap spectroscopy technique, which brought the system to the state $|e, e\rangle$. However, in [25] only the right avoided level crossing was examined. In contrast, here we predict an entirely inverted S-shaped signal (น) for the $|e, e\rangle$ initial state.

Focusing our attention on the insets of Figs. 3, 4, and 5 , where $g_{\perp} \sim 0.14 \mathrm{GHz}$. The transition energies, denoted by the black dashed lines, are the same in all of them. However, the corresponding spectral response differs significantly. For example, the EW spectrum in the inset of Fig. 3 tends to follow the TLS1's (horizontal) frequency, while for the inset of Fig. 4, it follows the TLS2's (hyperbolic) frequency. Quite different is the behavior presented in Fig. 5, where the spectral response near the avoided level crossing looks almost symmetric. The conspicuousness of the avoided level crossing (known as Rabi splitting) in such spectral responses confirms the fact that the TLS's interplay falls into the strong-coupling regime, i.e., the regime where the coupling strength, $g_{\perp}$, exceeds any individual decoherence rate of both TLS1 and TLS2; for all these cases, $g_{\perp} \gg \Gamma$. Indeed, in an experimental setting, the FWHM of each Lorentzian in the Rabi splitting can be considered proportional to the system's dissipation and decoherence rates. Therefore, we may incorporate some dissipation effects in the TLS spectra by tailoring the value of $\Gamma$.

TLS can interact not only with their hosting device but also with their own environment [21]. The coupling to phonon modes is the canonical source of dissipation for TLS [13]. Besides, the relaxation of TLS can also be due to their interaction with conduction electrons [48] and Bogoliubov quasiparticles [45]. However, their coupling 
to other low-frequency TLS, known as thermal fluctuators, is of utmost importance because it causes incoherent state switching and spectral diffusion in the TLS [49]. In several experiments, this decoherence mechanism produces spectroscopic signals of the TLS resonance frequency displaying telegraphic fluctuations [21, 25, 50]. Remarkably, with the analytical spectra here obtained, we can easily reproduce such effect by adding random fluctuations of a few $\mathrm{MHz}$ to $\bar{\Omega}_{k, 1}$. We show this in Fig. 7, where the resonance frequency of the TLS2 (gray dashedline) displays telegraphic fluctuations of $\sim \pm 10 \mathrm{MHz}[50]$. For comparison with a similar but experimental telegraphic signal, see Supplementary Fig. 1.c of [25].

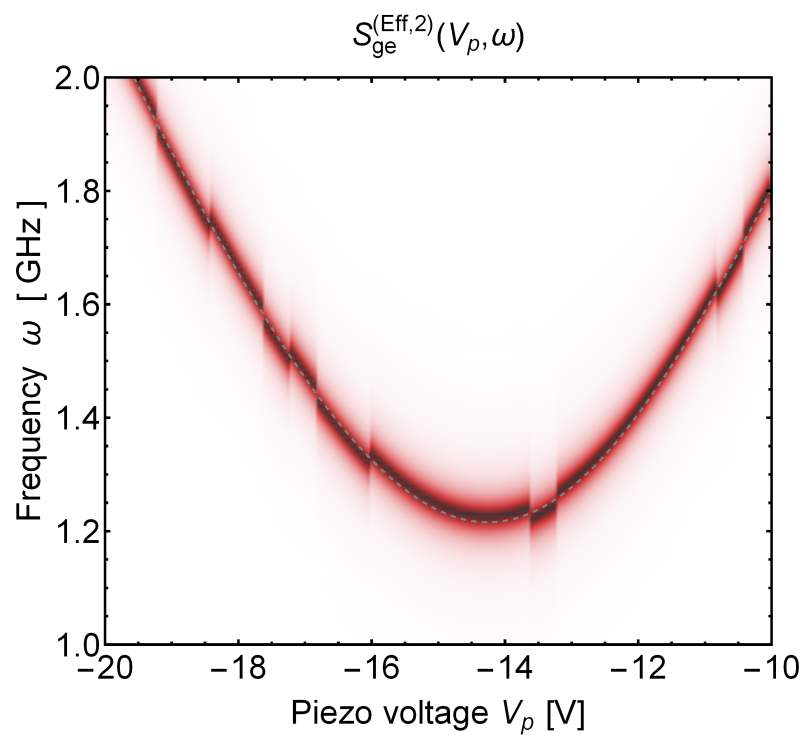

FIG. 7. Typical telegraphic signal of the TLS2 resonance frequency. This behavior is due to the interaction of TLS2 with a dominant low-frequency TLS (thermal fluctuator). The system parameters are the same as in Fig. 4, but for a plotted region near the symmetry point of TLS2.

We note in passing that, in the context of strain tuning experiments [19], the visibility of the spectral lines also depends on the coupling between each TLS with the superconducting qubit used as a detector. Thus, in principle, we could refine our results by considering such coupling. To do that, we need to include in (5) the interaction Hamiltonian given in the Supplementary Note 3 of [25]; however, this goes beyond the central goal of this work.

Overall, we consider that our propounded algebraic procedure (including the effective Hamiltonian model and results that follow from it) is efficient in terms of its algebraic simplicity and its capability to offer a reliable insight about an experimentalist is observing or might expect to observe under certain conditions, like those encounter in the weak or strong coupling regime. Furthermore, the theory incorporates parameter settings that can be adjusted at will (for instance, the applied piezo voltage) and allows to explore and identify possible effects of the strain upon the measurements, in terms of the semiquantitative spectrum provided by the theoretical model. Our findings reveal that one is, in principle, capable of identifying, on the basis of the comparison between the theoretical and experimental outcomes, the initial state of the system and the coupling regime of TLS-TLS interplay, as to, for instance, whether or not the counter-rotating terms of the interaction Hamiltonian should be kept; we determined that this is not the case. Instead, the model was recast to a more straightforward and effective Hamiltonian that only retains energypreserving rotating terms, which makes, in the strong coupling interaction case, the interpretation of the results simpler based upon the well-known rotating-wave approximation viewpoint.

\section{CONCLUSIONS}

We have proposed a simple description of spectral signatures of strongly interacting TLS located inside the tunnel barrier of JJs of superconducting qubits. The proposal is based upon the standard atomic tunneling model to analyze the interplay of coupled defects viewed as dipole-dipole interacting TLS. In the strong coupling regime, analytical expressions for the energy and power spectrum of two coherently interacting TLS are obtained via the full Lisenfeld model. Using a small rotation [30], we show that, for moderate coupling, a further simplified version of it, an effective model (8), turns out to offer an accurate replication of the outcomes derived from spectroscopic experimental implementations [25]. The analytical description of coupled defects relies on the employment of the physical spectrum of Eberly-Wódkiewicz by variation of their asymmetry energy $\varepsilon$, which depends linearly upon mechanical strain stemming from bending the chip circuit. The strain-tuning spectroscopy thus characterized can reveal the experimentally observable Sshaped frequency shift, and the expected level repulsion manifested as avoided level crossings, ascribable to strong defects' mutual dipole coupling, in addition to allowing us to trace the probability of the defect to be in a given initial state. Hopefully, experimentalists may adopt this approach, valid for any initial state of the composite system, as a supplemental tool to boost current studies of individual TLS. For instance, the EW spectrum could envision different types of Hamiltonians, describing unexplored spectral signatures of coupled TLS within disordered solids through strain-tuning spectroscopy. Furthermore, extractable information from this kind of spectroscopic explorations has become of particular relevance, in the context of quantum computers' performance, with a view to creating superconducting qubits of optimal functionality made up of low-loss insulators.

Disclosures. The authors declare no conflicts of interest.

Data availability statement. All data that support the findings of this study are included within the article. 
[1] A. Wallraff, D. I. Schuster, A. Blais, L. Frunzio, R.. S. Huang, J. Majer, S. Kumar, S. M. Girvin, and R. J. Schoelkopf, "Strong coupling of a single photon to a superconducting qubit using circuit quantum electrodynamics," Nature 431, 162-167 (2004).

[2] Frank Arute et al, "Quantum supremacy using a programmable superconducting processor," Nature 574, 505-510 (2019).

[3] Alexandre Blais, Arne L. Grimsmo, S. M. Girvin, and Andreas Wallraff, "Circuit quantum electrodynamics," Rev. Mod. Phys. 93, 025005 (2021).

[4] Aaron Somoroff, Quentin Ficheux, Raymond A. Mencia, Haonan Xiong, Roman V. Kuzmin, and Vladimir E. Manucharyan, "Millisecond coherence in a superconducting qubit," (2021), arXiv:2103.08578 [quant-ph].

[5] John M. Martinis, "Saving superconducting quantum processors from decay and correlated errors generated by gamma and cosmic rays," npj Quantum Information 7, 90 (2021).

[6] L. Cardani et al, "DEMETRA: Suppression of the relaxation induced by radioactivity in superconducting qubits," Journal of Low Temperature Physics 199, 475481 (2020).

[7] Conal E. Murray, "Material matters in superconducting qubits," Materials Science and Engineering: R: Reports 146, 100646 (2021).

[8] R. W. Simmonds, K. M. Lang, D. A. Hite, S. Nam, D. P. Pappas, and John M. Martinis, "Decoherence in Josephson phase qubits from junction resonators," Phys. Rev. Lett. 93, 077003 (2004).

[9] John M. Martinis, K. B. Cooper, R. McDermott, Matthias Steffen, Markus Ansmann, K. D. Osborn, K. Cicak, Seongshik Oh, D. P. Pappas, R. W. Simmonds, and Clare C. Yu, "Decoherence in Josephson qubits from dielectric loss," Phys. Rev. Lett. 95, 210503 (2005).

[10] Alexander L Burin, Andrii O Maksymov, and Kevin D Osborn, "Quantum coherent manipulation of two-level systems in superconducting circuits," Superconductor Science and Technology 27, 084001 (2014).

[11] Vassiliy Lubchenko and Peter G. Wolynes, "The microscopic quantum theory of low temperature amorphous solids," in Advances in Chemical Physics (John Wiley \& Sons, Ltd, 2007) Chap. 3, pp. 95-206.

[12] Anthony J. Leggett and Dervis C. Vural, "Tunneling twolevel systems model of the low-temperature properties of glasses: Are "Smoking-Gun" tests possible?" The Journal of Physical Chemistry B 117, 12966-12971 (2013), pMID: 23924397.

[13] Pablo Esquinazi, Tunneling systems in amorphous and crystalline solids (Springer Science \& Business Media, 2013).

[14] Clare C. Yu, "Why study noise due to two level systems: A suggestion for experimentalists," Journal of Low Temperature Physics 137, 251-265 (2004).

[15] A. Lupaşcu, P. Bertet, E. F. C. Driessen, C. J. P. M. Harmans, and J. E. Mooij, "One- and two-photon spectroscopy of a flux qubit coupled to a microscopic defect," Phys. Rev. B 80, 172506 (2009).

[16] A. Osman, J. Simon, A. Bengtsson, S. Kosen, P. Krantz, D. P. Lozano, M. Scigliuzzo, P. Delsing, Jonas Bylander, and A. Fadavi Roudsari, "Simplified Josephson-junction fabrication process for reproducibly high-performance superconducting qubits," Applied Physics Letters 118, 064002 (2021).

[17] Alexander Bilmes, Alexander K Händel, Serhii Volosheniuk, Alexey V Ustinov, and Jürgen Lisenfeld, "In-situ bandaged Josephson junctions for superconducting quantum processors," Superconductor Science and Technology 34, 125011 (2021).

[18] C T Earnest, J H Béjanin, T G McConkey, E A Peters, A Korinek, H Yuan, and M Mariantoni, "Substrate surface engineering for high-quality silicon/aluminum superconducting resonators," Superconductor Science and Technology 31, 125013 (2018).

[19] Grigorij J. Grabovskij, Torben Peichl, Jürgen Lisenfeld, Georg Weiss, and Alexey V. Ustinov, "Strain tuning of individual atomic tunneling systems detected by a superconducting qubit," Science 338, 232-234 (2012).

[20] Alexander Bilmes, Serhii Volosheniuk, Jan David Brehm, Alexey V. Ustinov, and Jürgen Lisenfeld, "Quantum sensors for microscopic tunneling systems," npj Quantum Information 7, 27 (2021).

[21] Clemens Müller, Jared H Cole, and Jürgen Lisenfeld, "Towards understanding two-level-systems in amorphous solids: insights from quantum circuits," Reports on Progress in Physics 82, 124501 (2019).

[22] A. M. Zagoskin, S. Ashhab, J. R. Johansson, and Franco Nori, "Quantum two-level systems in Josephson junctions as naturally formed qubits," Phys. Rev. Lett. 97, 077001 (2006).

[23] Matthew Neeley, M. Ansmann, Radoslaw C. Bialczak, M. Hofheinz, N. Katz, Erik Lucero, A. O'Connell, H. Wang, A. N. Cleland, and John M. Martinis, "Process tomography of quantum memory in a Josephson-phase qubit coupled to a two-level state," Nature Physics 4, 523-526 (2008).

[24] Alexander L. Burin, Douglas Natelson, Douglas D. Osheroff, and Yuri Kagan, "Interactions between tunneling defects in amorphous solids," in Tunneling Systems in Amorphous and Crystalline Solids, edited by Pablo Esquinazi (Springer Berlin Heidelberg, Berlin, Heidelberg, 1998) pp. 223-315.

[25] Jürgen Lisenfeld, Grigorij J. Grabovskij, Clemens Müller, Jared H. Cole, Georg Weiss, and Alexey V. Ustinov, "Observation of directly interacting coherent two-level systems in an amorphous material," Nature Communications 6, 6182 (2015).

[26] Jürgen Lisenfeld, Alexander Bilmes, Anthony Megrant, Rami Barends, Julian Kelly, Paul Klimov, Georg Weiss, John M. Martinis, and Alexey V. Ustinov, "Electric field spectroscopy of material defects in transmon qubits," npj Quantum Information 5, 105 (2019).

[27] Alexander Bilmes, Anthony Megrant, Paul Klimov, Georg Weiss, John M. Martinis, Alexey V. Ustinov, and Jürgen Lisenfeld, "Resolving the positions of defects in superconducting quantum bits," Scientific Reports 10, 3090 (2020).

[28] Alexander Bilmes, Resolving locations of defects in superconducting transmon qubits, Ph.D. thesis, Karlsruher Institut für Technologie (KIT) (2019).

[29] Alexander Bilmes, Serhii Volosheniuk, Alexey V. Ustinov, and Jürgen Lisenfeld, "Probing defect densities at 
the edges and inside Josephson junctions of superconducting qubits," (2021), arXiv:2108.06555 [quant-ph].

[30] A. B. Klimov and L. L. Sanchez-Soto, "Method of small rotations and effective hamiltonians in nonlinear quantum optics," Phys. Rev. A 61, 063802 (2000).

[31] A. B. Klimov, L. L. Sánchez-Soto, A. Navarro, and E. C. Yustas, "Effective Hamiltonians in quantum optics: a systematic approach," Journal of Modern Optics 49, 2211-2226 (2002).

[32] J H Eberly and K Wódkiewicz, "The time-dependent physical spectrum of light*," J. Opt. Soc. Am. 67, 12521261 (1977).

[33] J.D. Cresser, "Theory of the spectrum of the quantised light field," Physics Reports 94, 47-110 (1983).

[34] M Salado-Mejía, R Román-Ancheyta, F Soto-Eguibar, and H M Moya-Cessa, "Spectroscopy and critical quantum thermometry in the ultrastrong coupling regime," Quantum Science and Technology 6, 025010 (2021).

[35] R. Román-Ancheyta, O. de los Santos-Sánchez, L. Horvath, and H. M. Castro-Beltrán, "Time-dependent spectra of a three-level atom in the presence of electron shelving," Phys. Rev. A 98, 013820 (2018).

[36] Ricardo Román-Ancheyta, Barış Çakmak, and Özgür E Müstecaplıŏlu, "Spectral signatures of non-thermal baths in quantum thermalization," Quantum Science and Technology 5, 015003 (2020).

[37] W. A. Phillips, "Tunneling states in amorphous solids," Journal of Low Temperature Physics 7, 351-360 (1972).

[38] P. W. Anderson, B. I. Halperin, and C. M. Varma, "Anomalous low-temperature thermal properties of glasses and spin glasses," The Philosophical Magazine: A Journal of Theoretical Experimental and Applied Physics 25, 1-9 (1972).

[39] Clare C. Yu and Hervé M. Carruzzo, "Two-level systems and the tunneling model: A critical view," (2021), arXiv:2101.02787 [cond-mat.dis-nn].

[40] Michael W. Klein, Baruch Fischer, A. C. Anderson, and P. J. Anthony, "Strain interactions and the lowtemperature properties of glasses," Phys. Rev. B 18, 5887-5891 (1978).

[41] Anton Frisk Kockum, Adam Miranowicz, Simone De
Liberato, Salvatore Savasta, and Franco Nori, "Ultrastrong coupling between light and matter," Nature Reviews Physics 1, 19-40 (2019), 1807.11636.

[42] Stefano Scali, Janet Anders, and Luis A. Correa, "Local master equations bypass the secular approximation," Quantum 5, 451 (2021).

[43] Marco Cattaneo, Gian Luca Giorgi, Sabrina Maniscalco, and Roberta Zambrini, "Local versus global master equation with common and separate baths: superiority of the global approach in partial secular approximation," New Journal of Physics 21, 113045 (2019).

[44] Jürgen Lisenfeld, Alexander Bilmes, Shlomi Matityahu, Sebastian Zanker, Michael Marthaler, Moshe Schechter, Gerd Schön, Alexander Shnirman, Georg Weiss, and Alexey V. Ustinov, "Decoherence spectroscopy with individual two-level tunneling defects," Scientific Reports 6, 23786 (2016).

[45] Alexander Bilmes, Sebastian Zanker, Andreas Heimes, Michael Marthaler, Gerd Schön, Georg Weiss, Alexey V. Ustinov, and Jürgen Lisenfeld, "Electronic decoherence of two-level systems in a Josephson junction," Phys. Rev. B 96, 064504 (2017).

[46] J. Onam González, Luis A. Correa, Giorgio Nocerino, José P. Palao, Daniel Alonso, and Gerardo Adesso, "Testing the validity of the 'local' and 'global' GKLS master equations on an exactly solvable model," Open Systems \& Information Dynamics 24, 1740010 (2017).

[47] J.H. Eberly, C.V. Kunasz, and K. Wódkiewicz, "Timedependent spectrum of resonance fluorescence," Journal of Physics B-Atomic and Molecular Physics 13, 217-239 (1980).

[48] J. L. Black and P. Fulde, "Influence of the superconducting state upon the low-temperature properties of metallic glasses," Phys. Rev. Lett. 43, 453-456 (1979).

[49] Shlomi Matityahu, Alexander Shnirman, Gerd Schön, and Moshe Schechter, "Decoherence of a quantum twolevel system by spectral diffusion," Phys. Rev. B 93, 134208 (2016).

[50] Saskia M. Meißner, Arnold Seiler, Jürgen Lisenfeld, Alexey V. Ustinov, and Georg Weiss, "Probing individual tunneling fluctuators with coherently controlled tunneling systems," Phys. Rev. B 97, 180505 (2018). 